\title{
GENERATING SERIES FOR NETWORKS OF CHEN-FLIESS SERIES
}

\author{
W. STEVEN GRAY AND KURUSCH EBRAHIMI-FARD
}

\begin{abstract}
Consider a set of single-input, single-output nonlinear systems whose inputoutput maps are described only in terms of convergent Chen-Fliess series without any assumption that finite dimensional state space models are available. It is shown that any additive or multiplicative interconnection of such systems always has a Chen-Fliess series representation that can be computed explicitly in terms of iterated formal Lie derivatives.
\end{abstract}

\section{Contents}

1. Introduction 1

2. Preliminaries 2

3. Formal Realizations 4

4. Formal Representations 10

5. Networks of Chen-Fliess Series 13

6. Conclusions and Future Work 17

Acknowledgments 17

References

\section{INTRODUCTION}

The study of interconnections of nonlinear control systems is normally posed in a state space setting. Issues like controllability, observability and synchronization are natural to consider in this context [3, 23]. The goal of this paper is to consider networks of nonlinear systems described only in terms of Chen-Fliess series without any assumption that finite dimensional state space models are available [5, 6]. Such models are useful in the context of system identification as relatively few parameters need to be estimated to yield an accurate approximation of the input-output map [9, 21]. On the other hand, it is not automatically evident that any interconnection of such systems has a Chen-Fliess series representation. Dynamic output feedback systems, for example, where both the plant and controller have Chen-Fliess series representations have been shown to always have such a representation [7, 8]. The proof relies on the contraction mapping theorem applied in the ultrametric space of noncommutative formal power series. While a perfectly valid approach, it does not scale easily to complex networks. So in this paper an entirely different approach is taken based on the notion of a universal control system due to Kawski and Sussmann [14]. The idea is relatively straightforward in that networks of universal control systems are synthesized leading to the notion of a formal realization evolving on an $n$-fold direct product of formal Lie 
groups. Then the generating series for any input-output pair in the network is described using the notion of a formal representation, a type of infinite dimensional analogue of differential representations that are common in nonlinear control theory [13, 16]. It should be stated, however, that this does not prove that the resulting Chen-Fliess series converges in any sense. The tools used here are purely formal and algebraic. As is often the case when working with Chen-Fliess series, the algebra and the analytic issues can be considered separately with the former providing the setting for the latter, which is actually quite convenient [20]. In particular, it will be shown that any additive or multiplicative interconnection of a set of convergent single-input, single-output Chen-Fliess series always has a Chen-Fliess series representation that can be computed explicitly in terms of iterated formal Lie derivatives. The problem of determining convergence of the network's generating series will be deferred to future work.

The paper is organized as follows. The next section establishes the notation and terminology of the paper. Section 3 presents the concept of a formal realization. Formal representations are described in the subsequent section. The main results of the paper along with several examples are given in Section 5. The conclusions are summarized in the final section, as well as directions for future research.

\section{Preliminaries}

An alphabet $X=\left\{x_{0}, x_{1}, \ldots, x_{m}\right\}$ is any nonempty and finite set of noncommuting symbols referred to as letters. A word $\eta=x_{i_{1}} \cdots x_{i_{k}}$ is a finite sequence of letters from $X$. The number of letters in a word $\eta$, written as $|\eta|$, is called its length. The empty word, $\emptyset$, is taken to have length zero. The collection of all words having length $k$ is denoted by $X^{k}$. Define $X^{*}=\bigcup_{k>0} X^{k}$, which is a monoid under the concatenation (Cauchy) product. Any mapping $c: X^{*} \rightarrow \mathbb{R}^{\ell}$ is called a formal power series. Often $c$ is written as the formal sum $c=\sum_{\eta \in X^{*}}\langle c, \eta\rangle \eta$, where the coefficient $\langle c, \eta\rangle \in \mathbb{R}^{\ell}$ is the image of $\eta \in X^{*}$ under $c$. The support of $c, \operatorname{supp}(c)$, is the set of all words having nonzero coefficients. The set of all noncommutative formal power series over the alphabet $X$ is denoted by $\mathbb{R}^{\ell}\langle\langle X\rangle\rangle$. The subset of series with finite support, i.e., polynomials, is represented by $\mathbb{R}^{\ell}\langle X\rangle$. For any $c, d \in \mathbb{R}\langle\langle X\rangle\rangle$, the scalar product is $\langle c, d\rangle:=\sum_{\eta \in X^{*}}\langle c, \eta\rangle\langle d, \eta\rangle$, provided the sum is finite. The set $\mathbb{R}^{\ell}\langle\langle X\rangle\rangle$ is an associative $\mathbb{R}$-algebra under the concatenation product and an associative and commutative $\mathbb{R}$-algebra under the shuffle product, that is, the bilinear product uniquely specified by the shuffle product of two words

$$
\left(x_{i} \eta\right) \sqcup\left(x_{j} \xi\right)=x_{i}\left(\eta ш\left(x_{j} \xi\right)\right)+x_{j}\left(\left(x_{i} \eta\right) \uplus \xi\right),
$$

where $x_{i}, x_{j} \in X, \eta, \xi \in X^{*}$ and with $\eta ш \emptyset=\emptyset ш \eta=\eta[5]$. For any letter $x_{i} \in X$, let $x_{i}^{-1}$ denote the $\mathbb{R}$-linear left-shift operator defined by $x_{i}^{-1}(\eta)=\eta^{\prime}$ when $\eta=x_{i} \eta^{\prime}$ and zero otherwise. It acts as a derivation on the shuffle product. The Lie bracket $\left[x_{i}^{-1}, x_{j}^{-1}\right]=$ $x_{i}^{-1} x_{j}^{-1}-x_{j}^{-1} x_{i}^{-1}$ also acts as a derivation on the shuffle product. Finally, the left-shift operator is defined inductively for higher order shifts via $\left(x_{i} \eta\right)^{-1}=\eta^{-1} x_{i}^{-1}$, where $\eta \in X^{*}$. For $p \in \mathbb{R}\langle X\rangle$, let $p^{-1}:=\sum_{\eta \in X^{*}}\langle p, \eta\rangle \eta^{-1}$. 
Given any $c \in \mathbb{R}^{\ell}\langle\langle X\rangle\rangle$ one can associate a causal $m$-input, $\ell$-output operator, $F_{c}$, in the following manner. Let $\mathfrak{p} \geq 1$ and $t_{0}<t_{1}$ be given. For a Lebesgue measurable function $u:\left[t_{0}, t_{1}\right] \rightarrow \mathbb{R}^{m}$, define $\|u\|_{\mathfrak{p}}=\max \left\{\left\|u_{i}\right\|_{\mathfrak{p}}: 1 \leq i \leq m\right\}$, where $\left\|u_{i}\right\|_{\mathfrak{p}}$ is the usual $L_{\mathfrak{p}}$-norm for a measurable real-valued function, $u_{i}$, defined on $\left[t_{0}, t_{1}\right]$. Let $L_{\mathfrak{p}}^{m}\left[t_{0}, t_{1}\right]$ denote the set of all measurable functions defined on $\left[t_{0}, t_{1}\right]$ having a finite $\|\cdot\|_{\mathfrak{p}}$ norm and $B_{\mathfrak{p}}^{m}(R)\left[t_{0}, t_{1}\right]:=\{u \in$ $\left.L_{\mathfrak{p}}^{m}\left[t_{0}, t_{1}\right]:\|u\|_{\mathfrak{p}} \leq R\right\}$. Assume $C\left[t_{0}, t_{1}\right]$ is the subset of continuous functions in $L_{1}^{m}\left[t_{0}, t_{1}\right]$. Define inductively for each word $\eta=x_{i} \bar{\eta} \in X^{*}$ the map $E_{\eta}: L_{1}^{m}\left[t_{0}, t_{1}\right] \rightarrow C\left[t_{0}, t_{1}\right]$ by setting $E_{\emptyset}[u]=1$ and letting

$$
E_{x_{i} \bar{\eta}}[u]\left(t, t_{0}\right)=\int_{t_{0}}^{t} u_{i}(\tau) E_{\bar{\eta}}[u]\left(\tau, t_{0}\right) d \tau
$$

where $x_{i} \in X, \bar{\eta} \in X^{*}$, and $u_{0}=1$. The Chen-Fliess series corresponding to $c \in \mathbb{R}^{\ell}\langle\langle X\rangle\rangle$ is

$$
y(t)=F_{c}[u](t)=\sum_{\eta \in X^{*}}\langle c, \eta\rangle E_{\eta}[u]\left(t, t_{0}\right)
$$

[5]. If there exist real numbers $K_{c}, M_{c}>0$ such that

$$
|\langle c, \eta\rangle| \leq K_{c} M_{c}^{|\eta|}|\eta| !, \quad \forall \eta \in X^{*},
$$

then $F_{c}$ constitutes a well defined mapping from $B_{\mathfrak{p}}^{m}(R)\left[t_{0}, t_{0}+T\right]$ into $B_{\mathfrak{q}}^{\ell}(S)\left[t_{0}, t_{0}+T\right]$ for sufficiently small $R, T>0$ and some $S>0$, where the numbers $\mathfrak{p}, \mathfrak{q} \in[1, \infty]$ are conjugate exponents, i.e., $1 / \mathfrak{p}+1 / \mathfrak{q}=1[10]$. (Here, $|z|:=\max _{i}\left|z_{i}\right|$ when $z \in \mathbb{R}^{\ell}$.) The set of all such locally convergent series is denoted by $\mathbb{R}_{L C}^{\ell}\langle\langle X\rangle\rangle$, and $F_{c}$ is referred to as a Fliess operator.

Given Fliess operators $F_{c}$ and $F_{d}$, where $c, d \in \mathbb{R}_{L C}^{\ell}\langle\langle X\rangle\rangle$, the parallel and product connections satisfy $F_{c}+F_{d}=F_{c+d}$ and $F_{c} F_{d}=F_{c \omega d}$, respectively [5]. When Fliess operators $F_{c}$ and $F_{d}$ with $c \in \mathbb{R}_{L C}^{\ell}\langle\langle X\rangle\rangle$ and $d \in \mathbb{R}_{L C}^{m}\langle\langle X\rangle\rangle$ are interconnected in a cascade fashion, the composite system $F_{c} \circ F_{d}$ has the Fliess operator representation $F_{c o d}$, where the composition product of $c$ and $d$ is given by

$$
c \circ d=\sum_{\eta \in X^{*}}\langle c, \eta\rangle \psi_{d}(\eta)(\mathbf{1})
$$

[4]. Here 1 denotes the monomial $1 \emptyset$, and $\psi_{d}$ is the continuous (in the ultrametric sense) algebra homomorphism from $\mathbb{R}\langle\langle X\rangle\rangle$ to the vector space endomorphisms on $\mathbb{R}\langle\langle X\rangle\rangle, \operatorname{End}(\mathbb{R}\langle\langle X\rangle\rangle)$, uniquely specified by $\psi_{d}\left(x_{i} \eta\right)=\psi_{d}\left(x_{i}\right) \circ \psi_{d}(\eta)$ with $\psi_{d}\left(x_{i}\right)(e)=x_{0}\left(d_{i} ш e\right), i=0,1, \ldots, m$ for any $e \in \mathbb{R}\langle\langle X\rangle\rangle$, and where $d_{i}$ is the $i$-th component series of $d\left(d_{0}:=\mathbf{1}\right)$. By definition, $\psi_{d}(\emptyset)$ is the identity map on $\mathbb{R}\langle\langle X\rangle\rangle$. It is sometimes useful to associate a unique alphabet with each operator. For example, let $X=\left\{x_{0}, x_{1}, \ldots, x_{m}\right\}$ and $\tilde{X}=\left\{\tilde{x}_{0}, \tilde{x}_{1}, \ldots, \tilde{x}_{\tilde{m}}\right\}$. If $c \in \mathbb{R}_{L C}^{\tilde{\ell}}\langle\langle\tilde{X}\rangle\rangle$ and $d \in \mathbb{R}_{L C}^{\tilde{m}}\langle\langle X\rangle\rangle$, then the cascade connection $F_{c} \circ F_{d}$ has the generating series in $\mathbb{R}^{\tilde{\ell}}\langle\langle X\rangle\rangle$

$$
c \circ d=\sum_{\tilde{\eta} \in \tilde{X}^{*}}\langle c, \tilde{\eta}\rangle \psi_{d}(\tilde{\eta})(\mathbf{1}),
$$

where now $\psi_{d}\left(\tilde{x}_{i}\right): \mathbb{R}\langle\langle X\rangle\rangle \rightarrow \mathbb{R}\langle\langle X\rangle\rangle, e \mapsto x_{0}\left(d_{i} ш e\right), i=0,1, \ldots, \tilde{m}$. In this case, the letters in $X$ are identified with the inputs of $F_{d}$, and the letters of $\tilde{X}$ are identified with the inputs of $F_{c}$. There is a natural isomorphism between $x_{0}$ and $\tilde{x}_{0}$ since both symbols correspond to the unity input $\left(\tilde{u}_{0}=u_{0}=1\right)$. 
Example 2.1. Suppose $X=\left\{x_{0}, x_{1}\right\}$ and $\tilde{X}=\left\{\tilde{x}_{0}, \tilde{x}_{1}\right\}$. Let $c=\tilde{x}_{1} \tilde{x}_{1}$ and $d=x_{1}$. The generating series for the series interconnected system, $c \circ d=\tilde{x}_{1} \tilde{x}_{1} \circ x_{1}$, can be computed directly from $(2)$ as

$$
\begin{aligned}
c \circ d & =\left\langle c, \tilde{x}_{1} \tilde{x}_{1}\right\rangle \psi_{d}\left(\tilde{x}_{1} \tilde{x}_{1}\right)(\mathbf{1})=\psi_{d}\left(\tilde{x}_{1}\right) \circ \psi_{d}\left(\tilde{x}_{1}\right)(\mathbf{1}) \\
& =x_{0}\left(x_{1} \sqcup\left(x_{0}\left(x_{1} \sqcup \mathbf{1}\right)\right)\right)=x_{0} x_{1} x_{0} x_{1}+2 x_{0} x_{0} x_{1} x_{1} .
\end{aligned}
$$

It will be shown later (Examples 3.1 and 4.2) that this same result can be produced using formal realizations and formal representations.

\section{Formal Realizations}

For any finite $T>0, u \in L_{1}^{m}[0, T]$ and fixed $t \in[0, T]$, one can associate the formal power series in $\mathbb{R}\langle\langle X\rangle\rangle$

$$
P[u](t)=\sum_{\eta \in X^{*}} \eta E_{\eta}[u](t, 0)
$$

which is usually called a Chen series. If, for example, $u_{i}(t)=\alpha_{i} \in \mathbb{R}, i=1,2, \ldots, m$ on $[0, T]$ then $P[u](0)=\mathbf{1}$ and

$$
\begin{aligned}
\frac{d}{d t} P[u](t) & =\sum_{\eta \in X^{*}} \eta \frac{d}{d t} E_{\eta}[u](t, 0) \\
& =\sum_{\eta \in X^{*}} \sum_{i=0}^{m} \eta u_{i}(t) E_{x_{i}^{-1}(\eta)}[u](t, 0) \\
& =\sum_{\eta \in X^{*}} \sum_{i=0}^{m} \alpha_{i} x_{i} \eta E_{\eta}[u](t, 0) \\
& =\left(\sum_{i=0}^{m} \alpha_{i} x_{i}\right) P[u](t) .
\end{aligned}
$$

It follows directly that

$$
\frac{d^{n}}{d t^{n}} P[u](0)=\left(\sum_{i=0}^{m} \alpha_{i} x_{i}\right)^{n}, \quad n \geq 0
$$

and, therefore

$$
P[u](t)=\sum_{n=0}^{\infty}\left(\sum_{i=0}^{m} \alpha_{i} x_{i}\right)^{n} \frac{t^{n}}{n !}=\exp \left(t \sum_{i=0}^{m} \alpha_{i} x_{i}\right) .
$$

In general, $P[u]$ is the solution to the formal differential equation

$$
\frac{d}{d t} P[u]=\left(\sum_{i=0}^{m} x_{i} u_{i}\right) P[u], \quad P[u](0)=\mathbf{1},
$$

so that $P[u]$ is always the exponential of some Lie element over $X$. That is, if $\mathcal{L}(X)$ is the free Lie algebra generated by $X$, then any $d \in \mathbb{R}\langle\langle X\rangle\rangle$ is a Lie series if it can be written in the form $d=\sum_{n \geq 1} p_{n}$, where each polynomial $p_{n} \in \mathcal{L}(X)$ has support residing in $X^{n}$. The 
set of all Lie series will be denoted by $\widehat{\mathcal{L}}(X)$. An exponential Lie series is any series $e=$ $\exp (d):=\sum_{n>0} d^{n} / n$ !, where $d$ is a Lie series [18, Chapter 3]. In general, (3) has a solution of the form $P[u](t)=\exp (U(t))$ with $U(t) \in \widehat{\mathcal{L}}(X), t \geq 0$ [18, Corollary 3.5]. As a consequence of the Baker-Campbell-Hausdorff formula, which states that $\log \left(\exp \left(x_{i}\right) \exp \left(x_{j}\right)\right)$ is a Lie series, the set of all exponential Lie series forms a group, $\mathcal{G}(X)$, under the Cauchy product with unit 1 [2, Lemma 3] and [18, Corollary 3.3].

Following the approach of Kawski and Sussmann in [14, 19, $\mathcal{G}(X)$ can be viewed as a formal Lie group with $\widehat{\mathcal{L}}(X)$ as its corresponding Lie algebra ${ }^{1}$ A commutative algebra of real-valued functions on $\mathcal{G}(X)$ is defined using the shuffle algebra on the $\mathbb{R}$-vector space $\mathbb{R}_{L C}\langle\langle X\rangle\rangle$. Specifically, for any fixed $c \in \mathbb{R}_{L C}\langle\langle X\rangle\rangle$ define $f_{c}: \mathcal{G}(X) \rightarrow \mathbb{R}$ in terms of the scalar product as

$$
z \mapsto f_{c}(z)=\langle c, z\rangle=\sum_{\eta \in X^{*}}\langle c, \eta\rangle\langle z, \eta\rangle .
$$

Ree's criterion states that $p \in \mathcal{L}(X)$ if and only if $\langle\eta \omega \nu, p\rangle=0$ for all nonempty words $\eta, \nu \in X^{*}$ [Theorem 2.2][17]. This implies that $z$ is an exponential Lie series if and only if $\langle c \sqcup d, z\rangle=\langle c, z\rangle\langle d, z\rangle$ for all $c, d \in \mathbb{R}\langle\langle X\rangle\rangle$ [18, Theorem 3.2]. Therefore,

$$
f_{c}(z) f_{d}(z)=\langle c, z\rangle\langle d, z\rangle=\langle c \varpi d, z\rangle=f_{c \varpi d}(z) .
$$

Convergence follows from the fact that the shuffle product is known to preserve local convergence [22] 2] Often $f_{c}(z)$ will be abbreviated as $c(z)$, which is more natural in the present context. Analogous to standard Lie group theory, the formal tangent space at the unit $1, T_{1} \mathcal{G}(X)$, is identified with $\widehat{\mathcal{L}}(X)$. Thus, for any fixed $p \in \widehat{\mathcal{L}}(X)$, there is a corresponding tangent vector at $\mathbf{1}$ written as the linear functional $V_{p}(\mathbf{1}): \mathbb{R}_{L C}\langle\langle X\rangle\rangle \rightarrow \mathbb{R}$, $c \mapsto V_{p}(\mathbf{1})(c):=\langle c, p \mathbf{1}\rangle$ and satisfying the Leibniz rul $\rfloor^{3}$

$$
\begin{aligned}
V_{p}(\mathbf{1})(c \amalg d) & =\langle c \amalg d, p \mathbf{1}\rangle \\
& =\left\langle p^{-1}(c \amalg d), \mathbf{1}\right\rangle \\
& =\left\langle p^{-1}(c) \amalg d, \mathbf{1}\right\rangle+\left\langle c \varpi p^{-1}(d), \mathbf{1}\right\rangle \\
& =\left\langle p^{-1}(c), \mathbf{1}\right\rangle\langle d, \mathbf{1}\rangle+\langle c, \mathbf{1}\rangle\left\langle p^{-1}(d), \mathbf{1}\right\rangle \\
& =\langle c, p \mathbf{1}\rangle\langle d, \mathbf{1}\rangle+\langle c, \mathbf{1}\rangle\langle d, p \mathbf{1}\rangle \\
& =V_{p}(\mathbf{1})(c) d(\mathbf{1})+c(\mathbf{1}) V_{p}(\mathbf{1})(d) .
\end{aligned}
$$

In turn, the tangent space at $z \in \mathcal{G}(X)$, denoted $T_{z} \mathcal{G}(X)$, is defined via right translation to be the vector space of linear functionals $V_{p}(z): \mathbb{R}_{L C}\langle\langle X\rangle\rangle \rightarrow \mathbb{R}, c \mapsto V_{p}(z)(c):=\langle c, p z\rangle$, $p \in \widehat{\mathcal{L}}(X)$ satisfying

$$
\begin{aligned}
V_{p}(z)(c \varpi d) & =\langle c \varpi d, p z\rangle \\
& =\langle c, p z\rangle\langle d, z\rangle+\langle c, z\rangle\langle d, p z\rangle
\end{aligned}
$$

\footnotetext{
${ }^{1}$ Certain aspects of this framework can also be found in [11, 12.

${ }^{2}$ The authors of [14, 19] defined their algebra on $\mathbb{R}\langle X\rangle$, which entirely avoids the convergence issue. But here $\mathbb{R}_{L C}\langle\langle X\rangle\rangle$ is more suitable for the applications to follow.

${ }^{3}$ Recall the definition of the scalar product in the previous section.
} 


$$
=V_{p}(z)(c) d(z)+c(z) V_{p}(z)(d) .
$$

From a Hopf algebraic viewpoint [15], elements $z \in \mathcal{G}(X)$ are group-like, that is, for $c, d \in$ $\mathbb{R}_{L C}\langle\langle X\rangle\rangle$ one has $\langle c \amalg d, z\rangle=\left\langle c \otimes d, \Delta_{\uplus} z\right\rangle=\langle c \otimes d, z \otimes z\rangle=\langle c, z\rangle\langle d, z\rangle$. Here $\Delta_{\omega}$ is the unshuffle coproduct dualizing the shuffle product. On the other hand, elements $p \in \widehat{\mathcal{L}}(X)$ are primitive, i.e., $\Delta_{\omega} p=p \otimes \mathbf{1}+\mathbf{1} \otimes p$ such that $\langle c \varpi d, p\rangle=\langle c, p\rangle\langle d, \mathbf{1}\rangle+\langle c, \mathbf{1}\rangle\langle d, p\rangle$. Moreover, $\Delta_{\uplus} p z=\Delta_{ш} p \Delta_{\uplus} z$ yields $\langle c \amalg d, p z\rangle=\langle c, p z\rangle\langle d, z\rangle+\langle c, z\rangle\langle d, p z\rangle$. However, in this work a Hopf algebraic approach has been suppressed in favor of a purely Lie theoretic presentation.

For any $p \in \widehat{\mathcal{L}}(X)$, the mapping

$$
V_{p}: \mathcal{G}(X) \rightarrow T_{z} \mathcal{G}(X), z \mapsto V_{p}(z):=p z
$$

is a formal right-invariant vector field on $\mathcal{G}(X)$. Here $\mathcal{X}$ will denote the set of all such rightinvariant vector fields. In addition, the formal Lie derivative is defined to be the mapping

$$
L_{p}: \mathbb{R}_{L C}\langle\langle X\rangle\rangle \rightarrow \mathbb{R}_{L C}\langle\langle X\rangle\rangle, c \mapsto L_{p} c:=p^{-1} c
$$

so that

$$
L_{p} c(z)=\left\langle L_{p} c, z\right\rangle=\left\langle p^{-1} c, z\right\rangle=\langle c, p z\rangle=V_{p}(z)(c),
$$

and, in particular,

$$
\begin{aligned}
L_{p}(c \amalg d)(z) & =\left\langle L_{p}(c \amalg d), z\right\rangle \\
& =\langle c \amalg d, p z\rangle \\
& =\left(L_{p} c(z)\right) d(z)+c(z) L_{p} d(z),
\end{aligned}
$$

which is just an alternative form of (5).

Finally, note that (1) can be written componentwise as $y_{k}(t)=\left\langle c_{k}, z(t)\right\rangle, k=1,2, \ldots, \ell$, where $c_{k} \in \mathbb{R}_{L C}\langle\langle X\rangle\rangle$ denotes the $k$-th component of $c \in \mathbb{R}_{L C}^{\ell}\langle\langle X\rangle\rangle$ and $z(t)=P[u](t)$. This leads to the following definition.

Definition 3.1. For any $c \in \mathbb{R}_{L C}^{\ell}\langle\langle X\rangle\rangle$, the formal realization of the Fliess operator $y=F_{c}[u]$ is

$$
\begin{aligned}
\dot{z} & =\sum_{i=0}^{m} x_{i} z u_{i}, \quad z(0)=\mathbf{1} \\
y_{k} & =\left\langle c_{k}, z\right\rangle, \quad k=1,2, \ldots, \ell .
\end{aligned}
$$

Observe that

$$
\begin{aligned}
L_{x_{i}} c_{k}(\mathbf{1}) & =x_{i}^{-1} c_{k}(\mathbf{1})=\left\langle x_{i}^{-1} c_{k}, \mathbf{1}\right\rangle=\left\langle c_{k}, x_{i}\right\rangle \\
L_{x_{j}} L_{x_{i}} c_{k}(\mathbf{1}) & =x_{j}^{-1} x_{i}^{-1} c_{k}(\mathbf{1})=\left\langle x_{j}^{-1} x_{i}^{-1} c_{k}, \mathbf{1}\right\rangle=\left\langle c_{k}, x_{i} x_{j}\right\rangle,
\end{aligned}
$$

so that the coefficients of $c_{k}$ can always be written in terms of formal Lie derivatives as

$$
\begin{aligned}
\left\langle c_{k}, \eta\right\rangle & =\left\langle c_{k}, x_{i_{1}} \cdots x_{i_{k}}\right\rangle \\
& =L_{x_{i_{k}}} \cdots L_{x_{i_{1}}} c_{k}(\mathbf{1})=: L_{\eta} c_{k}(\mathbf{1}) .
\end{aligned}
$$


The notion of a formal realization in Definition 3.1 is now extended by taking a finite number of direct products of $\mathcal{G}(X)$, i.e., $\mathcal{G}^{n}(X):=\mathcal{G}(X) \times \mathcal{G}(X) \times \cdots \times \mathcal{G}(X)$, where $\mathcal{G}(X)$ appears $n$ times. For any $\hat{c}=c_{1} \otimes \cdots \otimes c_{n} \in \mathbb{R}_{L C}^{\otimes n}\langle\langle X\rangle\rangle$ define

$$
\begin{aligned}
& f_{\hat{c}}: \mathcal{G}^{n}(X) \rightarrow \mathbb{R} \\
& \quad z \mapsto\left(c_{1} \otimes \cdots \otimes c_{n}\right)\left(z_{1}, \ldots, z_{n}\right)=\left\langle c_{1}, z_{1}\right\rangle \cdots\left\langle c_{n}, z_{n}\right\rangle .
\end{aligned}
$$

A commutative algebra on the $\mathbb{R}$-vector space of all such real-valued functions on $\mathcal{G}^{n}(X)$ is given by defining

$$
\begin{aligned}
f_{\hat{c}}(z) f_{\hat{d}}(z) & =\left[\left\langle c_{1}, z_{1}\right\rangle \cdots\left\langle c_{n}, z_{n}\right\rangle\right]\left[\left\langle d_{1}, z_{1}\right\rangle \cdots\left\langle d_{n}, z_{n}\right\rangle\right] \\
& =\left\langle c_{1} \amalg d_{1}, z_{1}\right\rangle \cdots\left\langle c_{n} \uplus d_{n}, z_{n}\right\rangle \\
& =:(\hat{c} \amalg \hat{d})\left(z_{1}, z_{2}, \ldots, z_{n}\right) \\
& =f_{\hat{c} \amalg \hat{d}}(z) .
\end{aligned}
$$

As earlier, $f_{\hat{c}}(z)$ will often be abbreviated as $\hat{c}(z)$. The Lie algebra of $\mathcal{G}^{n}(X)$, denoted by $\widehat{\mathcal{L}}^{n}(X)$, is similarly defined as the $n$-fold direct sum of the Lie algebra $\widehat{\mathcal{L}}(X)$ for $\mathcal{G}(X)$ with itself. The formal tangent space at the unit $\mathbf{1}_{n}:=(\mathbf{1}, \ldots, \mathbf{1}), T_{\mathbf{1}_{n}} \mathcal{G}^{n}(X)$, is identified with $\widehat{\mathcal{L}}^{n}(X)$ via the one-parameter subgroup $H(t):=\left(\exp \left(t p_{1}\right), \exp \left(t p_{2}\right), \ldots, \exp \left(t p_{n}\right)\right), p=$ $\left(p_{1}, p_{2}, \ldots, p_{n}\right) \in \widehat{\mathcal{L}}^{n}(X)$ so that $\dot{H}(0)=p$. For any fixed $p \in \widehat{\mathcal{L}}^{n}(X)$, there is a corresponding tangent vector at $\mathbf{1}_{n}$ represented by the linear functional

$$
V_{p}\left(\mathbf{1}_{n}\right): \mathbb{R}_{L C}^{\otimes n}\langle\langle X\rangle\rangle \rightarrow \mathbb{R},\left.\hat{c} \mapsto \frac{d}{d t}(\hat{c} \circ H(t))\right|_{t=0} .
$$

Observe that

$$
\begin{aligned}
V_{p}\left(\mathbf{1}_{n}\right)(\hat{c}) & =\left.\frac{d}{d t}\left(\left\langle c_{1}, \exp \left(t p_{1}\right)\right\rangle \cdots\left\langle c_{i}, \exp \left(t p_{i}\right)\right\rangle \cdots\left\langle c_{n}, \exp \left(t p_{n}\right)\right\rangle\right)\right|_{t=0} \\
& =\sum_{i=1}^{n}\left\langle c_{1}, \mathbf{1}\right\rangle \cdots\left\langle c_{i}, p_{i} \mathbf{1}\right\rangle \cdots\left\langle c_{n}, \mathbf{1}\right\rangle
\end{aligned}
$$

satisfies the Leibniz rule:

$$
\begin{aligned}
& V_{p}\left(\mathbf{1}_{n}\right)(\hat{c} \amalg \hat{d})=\sum_{i=1}^{n}\left\langle c_{1} \uplus d_{1}, \mathbf{1}\right\rangle \cdots\left\langle c_{i} \uplus d_{i}, p_{i} \mathbf{1}\right\rangle \cdots\left\langle c_{n} ш d_{n}, \mathbf{1}\right\rangle \\
& =\sum_{i=1}^{n}\left\langle c_{1} ш d_{1}, \mathbf{1}\right\rangle \cdots\left\langle p_{i}^{-1}\left(c_{i} ш d_{i}\right), \mathbf{1}\right\rangle \cdots\left\langle c_{n} ш d_{n}, \mathbf{1}\right\rangle \\
& =\sum_{i=1}^{n}\left\langle c_{1} ш d_{1}, \mathbf{1}\right\rangle \cdots\left\langle p_{i}^{-1}\left(c_{i}\right) ш d_{i}, \mathbf{1}\right\rangle \cdots\left\langle c_{n} ш d_{n}, \mathbf{1}\right\rangle+ \\
& \left.\sum_{i=1}^{n}\left\langle c_{1} ш d_{1}, \mathbf{1}\right\rangle \cdots\left\langle c_{i} ш p_{i}^{-1}\left(d_{i}\right)\right), \mathbf{1}\right\rangle \cdots\left\langle c_{n} ш d_{n}, \mathbf{1}\right\rangle \\
& =V_{p}\left(\mathbf{1}_{n}\right)(\hat{c}) \hat{d}\left(\mathbf{1}_{n}\right)+\hat{c}\left(\mathbf{1}_{n}\right) V_{p}\left(\mathbf{1}_{n}\right)(\hat{d}) .
\end{aligned}
$$


The tangent space at $z \in \mathcal{G}^{n}(X)$, denoted $T_{z} \mathcal{G}^{n}(X)$, is defined via right translation to be the vector space of linear functionals

$$
\begin{aligned}
V_{p}(z): \mathbb{R}_{L C}^{\otimes n}\langle\langle X\rangle\rangle \rightarrow \mathbb{R} \\
\hat{c} \mapsto \sum_{i=1}^{n}\left\langle c_{1}, z_{1}\right\rangle \cdots\left\langle c_{i}, p_{i} z_{i}\right\rangle \cdots\left\langle c_{n}, z_{n}\right\rangle
\end{aligned}
$$

so as to satisfy

$$
V_{p}(z)(\hat{c} \amalg \hat{d})=V_{p}(z)(\hat{c}) \hat{d}(z)+\hat{c}(z) V_{p}(z)(\hat{d}) .
$$

For any $p \in \widehat{\mathcal{L}}^{n}(X)$, the mapping

$$
V_{p}: \mathcal{G}^{n}(X) \rightarrow T_{z} \mathcal{G}^{n}(X), z \mapsto\left(p_{1} z_{1}, \ldots, p_{n} z_{n}\right)
$$

is a formal right-invariant vector field on $\mathcal{G}^{n}(X)$. Here $\mathcal{X}^{n}$ will denote the set of all such right-invariant vector fields. In this context, the formal Lie derivative is defined to be the mapping

$$
\begin{aligned}
L_{p}: \mathbb{R}_{L C}^{\otimes n}\langle\langle X\rangle\rangle \rightarrow \mathbb{R}_{L C}^{\otimes n}\langle\langle X\rangle\rangle \\
c_{1} \otimes \cdots \otimes c_{n} \mapsto \sum_{i=1}^{n} c_{1} \otimes \cdots \otimes p_{i}^{-1}\left(c_{i}\right) \otimes \cdots \otimes c_{n}
\end{aligned}
$$

so that

$$
\begin{aligned}
L_{p} \hat{c}(z) & =\left(\sum_{i=1}^{n} c_{1} \otimes \cdots \otimes p_{i}^{-1}\left(c_{i}\right) \otimes \cdots \otimes c_{n}\right)\left(z_{1}, \ldots, z_{n}\right) \\
& =\sum_{i=1}^{n}\left\langle c_{1}, z_{1}\right\rangle \cdots\left\langle c_{i}, p_{i} z_{i}\right\rangle \cdots\left\langle c_{n}, z_{n}\right\rangle \\
& =V_{p}(z)(\hat{c}),
\end{aligned}
$$

and directly

$$
L_{p}(\hat{c} \amalg \hat{d})(z)=\left(L_{p} \hat{c}(z)\right) \hat{d}(z)+\hat{c}(z) L_{p} \hat{d}(z) .
$$

In this generalized setting, a set of $n$ systems with state $z=\left(z_{1}, z_{2}, \ldots, z_{n}\right)$ evolves on the group $\mathcal{G}^{n}(X)$ according to the formal state equations

$$
\dot{z}_{j}=\sum_{i=0}^{m} x_{i} z_{j} u_{i j}, \quad z_{j}(0)=\mathbf{1}
$$

where $u_{i j} \in L_{\mathfrak{p}}[0, T]$ and $u_{0 j}=1$ for $i=1,2, \ldots, m, j=1,2, \ldots, n$. Define $\ell$ outputs $y_{k}=\hat{c}_{k}(z)$, where $\hat{c}_{k} \in \mathbb{R}_{L C}^{\otimes n}\langle\langle X\rangle\rangle, k=1,2, \ldots, \ell$. Therefore, the corresponding input-output map $u \mapsto y$ takes an $m \times n$ matrix of inputs to $\ell$ outputs. Consider now the situation where a network is formed by allowing each system input to be interconnected to some function of other systems' outputs and a new external input $v_{i j}$ to yield a new input-output map $v \mapsto y$, for example, $u_{i j}=\hat{d}_{i j}(z)+v_{i j}$, where $\hat{d}_{i j} \in \mathbb{R}_{L C}^{\otimes n}\langle\langle X\rangle\rangle$. In this case, the state equations for the interconnected system become

$$
\dot{z}_{j}=x_{0} z_{j}+\sum_{i=1}^{m} x_{i} \hat{d}_{i j}(z) z_{j}+x_{i} z_{j} v_{i j}, \quad z_{j}(0)=\mathbf{1} .
$$


Note, in particular, the appearance of state dependent vector fields $p_{j} z_{j}$ with $p_{j}(t)=$ $\sum_{i=1}^{m} x_{i} \hat{d}_{i j}(z(t)) \in \widehat{\mathcal{L}}(X)$. The solution to $\dot{z}_{j}=p_{j} z_{j}, z_{j}(0)=\mathbf{1}$ has the form $z_{j}(t)=$ $\exp \left(U_{j}(t)\right)$, where $U_{j}(t) \in \widehat{\mathcal{L}}(X)$. The corresponding tangent vector at $z(t)$ is

$$
\begin{aligned}
V_{p(t)}(z(t)) & : \mathbb{R}_{L C}^{\otimes n}\langle\langle X\rangle\rangle \rightarrow \mathbb{R} \\
\hat{c} & \mapsto \frac{d}{d t}(\hat{c} \circ z(t)) \\
& =\sum_{j=1}^{n}\left\langle c_{1}, z_{1}(t)\right\rangle \cdots\left\langle c_{j}, p_{j}(t) z_{j}(t)\right\rangle \cdots\left\langle c_{n}, z_{n}(t)\right\rangle \\
& =L_{p(t)} \hat{c}(z(t)) .
\end{aligned}
$$

Substituting $p_{j}(t)=\sum_{i=1}^{m} x_{i} \hat{d}_{i j}(z(t))$ on the right-hand side above, where $\hat{d}_{i j}(z(t))=$ $\left\langle d_{i j}^{(1)}, z_{1}(t)\right\rangle \cdots\left\langle d_{i j}^{(n)}, z_{n}(t)\right\rangle$, gives

$$
\begin{aligned}
L_{p(t)} \hat{c}(z(t)) & =\sum_{j=1}^{n}\left\langle c_{1}, z_{1}(t)\right\rangle \cdots\left\langle c_{j}, p_{j}(t) z_{j}(t)\right\rangle \cdots\left\langle c_{n}, z_{n}(t)\right\rangle \\
& =\sum_{j=1}^{n}\left\langle c_{1}, z_{1}(t)\right\rangle \cdots \sum_{i=1}^{m} \hat{d}_{i j}(z(t))\left\langle c_{j}, x_{i} z_{j}(t)\right\rangle \cdots\left\langle c_{n}, z_{n}(t)\right\rangle \\
& =\sum_{i=1}^{m} \sum_{j=1}^{n}\left\langle c_{1} ш d_{i j}^{(1)}, z_{1}(t)\right\rangle \cdots\left\langle x_{i}^{-1}\left(c_{j} ш d_{i j}^{(j)}\right), z_{j}(t)\right\rangle \cdots\left\langle c_{n} \amalg d_{i j}^{(n)}, z_{n}(t)\right\rangle \\
& =: \hat{c}^{\prime}(z(t)) .
\end{aligned}
$$

In this way, a second Lie derivative can now be computed directly using (8), thus circumventing the difficult task of explicitly composing time-varying vector fields. Henceforth, all such state dependent Lie series will be written as $p(z)$. No other type of state dependent series will appear in this paper. In this context, a generalization of Definition 3.1 is presented.

Definition 3.2. Let $V_{i} \in \mathcal{X}^{n}, i=0,1, \ldots, m$ with

$$
\begin{aligned}
V_{i} & : \mathcal{G}^{n}(X) \rightarrow T_{z} \mathcal{G}^{n}(X) \\
& z=\left(z_{1}, \ldots, z_{n}\right) \mapsto V_{i}(z)=\left(V_{i 1}(z) z_{1}, \ldots, V_{i n}(z) z_{n}\right),
\end{aligned}
$$

where $V_{i j}(z(t)) \in \widehat{\mathcal{L}}(X)$. The $j$-th component of the corresponding state equation on $\mathcal{G}^{n}(X)$ is

$$
\dot{z}_{j}=\sum_{i=0}^{m} V_{i j}(z) z_{j} u_{i j}, \quad z_{j}(0)=z_{j 0}
$$

Given $\hat{c}_{k} \in \mathbb{R}_{L C}^{\otimes n}\langle\langle X\rangle\rangle, k=1,2, \ldots, \ell$, the $k$-th output equation is defined to be

$$
y_{k}=\hat{c}_{k}(z) \text {. }
$$

Collectively, $\left(V, z_{0}, \hat{c}\right)$ is a formal realization on $\mathcal{G}^{n}(X)$ of the formal input-output map $u \mapsto y$. 
For convenience the integer $n$ will be referred to here as the dimension of the realization, though this is a misnomer as the underlying group $\mathcal{G}(X)$ is not finite dimensional, therefore neither is the state $z$. The following example illustrates how the concept naturally arises when Chen-Fliess series are composed.

Example 3.1. Reconsider the systems $y_{2}=F_{c}\left[u_{2}\right]$ and $y_{1}=F_{d}\left[u_{1}\right]$ in Example 2.1 using the same alphabet $X=\left\{x_{0}, x_{1}\right\}$ for both series. Each has a formal realization of the form given in Definition 3.1. Setting $u_{2}=y_{1}$ so that $y_{2}=F_{c} \circ F_{d}\left[u_{1}\right]$ yields a formal realization of dimension two:

$$
\begin{aligned}
& \dot{z}_{1}=x_{0} z_{1}+x_{1} z_{1} u_{1}, \quad z_{1}(0)=\mathbf{1} \\
& \dot{z}_{2}=\left(x_{0}+x_{1}\left\langle d, z_{1}\right\rangle\right) z_{2}, \quad z_{2}(0)=\mathbf{1} \\
& y_{2}=\left\langle\mathbf{1}, z_{1}\right\rangle\left\langle c, z_{2}\right\rangle .
\end{aligned}
$$

(Note that $\left\langle\mathbf{1}, z_{1}\right\rangle=1$.) Therefore,

$$
V_{0}(z)=\left[\begin{array}{c}
x_{0} z_{1} \\
\left(x_{0}+x_{1}\left\langle d, z_{1}\right\rangle\right) z_{2}
\end{array}\right], \quad V_{1}(z)=\left[\begin{array}{c}
x_{1} z_{1} \\
0
\end{array}\right],
$$

and $\hat{c}=\mathbf{1} \otimes c$. Observe that the composition $F_{c} \circ F_{d}=F_{c o d}$ introduces in the second component of the tangent vector $V_{0}(z)$ a $z_{1}$ dependence. The aim is to express $c \circ d$ directly in terms of $\left(V, \mathbf{1}_{2}, \hat{c}\right)$. This leads to the notion of a formal representation of a series as presented in the next section. It can be viewed as a generalization of (6).

\section{Formal Representations}

The following definition is a formal analog of a differential representation as appears, for example, in [13, 16].

Definition 4.1. A formal representation of a series $d \in \mathbb{R}\langle\langle X\rangle\rangle$ is any triple $\left(\mu, z_{0}, \hat{c}\right)$, where

$$
\mu: X^{*} \rightarrow \mathcal{X}^{n}, x_{i} \mapsto V_{i}
$$

defines a monoid homomorphism, $z_{0} \in \mathcal{G}^{n}(X)$, and $\hat{c} \in \mathbb{R}_{L C}^{\otimes n}\langle\langle X\rangle\rangle$, so that for any word $\eta=x_{i_{k}} x_{i_{k-1}} \cdots x_{i_{1}} \in X^{*}$

$$
\langle d, \eta\rangle=L_{\mu(\eta)} \hat{c}\left(z_{0}\right):=L_{\mu\left(x_{i_{1}}\right)} L_{\mu\left(x_{i_{2}}\right)} \cdots L_{\mu\left(x_{i_{k}}\right)} \hat{c}\left(z_{0}\right) .
$$

By definition, $\langle d, \emptyset\rangle=L_{\emptyset} \hat{c}\left(z_{0}\right):=\hat{c}\left(z_{0}\right)$. The integer $n \geq 1$ will be called the dimension of the representation.

Example 4.1. For the trivial case where $n=1, \mu\left(x_{i}\right)=x_{i}, z_{0}=\mathbf{1}$, and $d=\hat{c}=c$ it is immediate that (12) reduces to $(6)$ with $\ell=1$.

The following lemma provides a sufficient condition under which formal representations are always well defined. 
Lemma 4.1. Given $\left(\mu, z_{0}, \hat{c}\right)$, if for each $x_{i} \in X\left[\mu\left(x_{i}\right)\right]_{j}(z):=V_{i j}(z) z_{j}$ with $V_{i j}(z)$ being some Lie polynomial in $\mathcal{L}(X)$, then there exists a well defined $d \in \mathbb{R}\langle\langle X\rangle\rangle$ satisfying (12).

Proof: If $\left(\mu, z_{0}, \hat{c}\right)$ is a formal representation of $d$ then necessarily for any $\eta=x_{i_{1}} \cdots x_{i_{k}} \in X^{*}$

$$
\left.\left\langle d, x_{i_{k}} \cdots x_{i_{1}}\right\rangle=L_{\mu\left(x_{i_{1}}\right)} L_{\mu\left(x_{i_{2}}\right)} \cdots L_{\mu\left(x_{i_{k}}\right)}\right) \hat{c}\left(z_{0}\right),
$$

where each $V_{i j}(z)$ is assumed to be a Lie polynomial. Therefore, each Lie derivative can be written as a polynomial in functions of the form $\left\langle e, p_{i} z_{i}\right\rangle$ with $p_{i} \in \mathcal{L}(X), i=1,2, \ldots, n$, and $e \in \mathbb{R}_{L C}\langle\langle X\rangle\rangle$, implying that $d$ is well defined, in fact, locally finite [1].

Example 4.2. Continuing Examples 2.1 and 3.1, the claim is that $c \circ d$ has a formal representation $\left(\mu, \mathbf{1}_{2}, \hat{c}\right)$, where $\mu$ is defined in terms of the vector fields $V_{0}$ and $V_{1}$ in Example 3.1 and $\hat{c}=\mathbf{1} \otimes c$. Note that both vector fields satisfy the condition in Lemma 4.1. As an example, it is verified that

$$
\left\langle x_{0}^{2} x_{1}^{2}, c \circ d\right\rangle=L_{\mu\left(x_{0}^{2} x_{1}^{2}\right)} \hat{c}(\mathbf{1})=L_{V_{1}} L_{V_{1}} L_{V_{0}} L_{V_{0}} \hat{c}(\mathbf{1})=2 .
$$

First apply (8) (suppressing all $t$ dependence)

$$
\begin{aligned}
L_{V_{0}} \hat{c}(z) & =\left\langle c, V_{02}(z) z_{2}\right\rangle \\
& =\left\langle x_{1}^{2},\left(x_{0}+x_{1}\left\langle x_{1}, z_{1}\right\rangle\right) z_{2}\right\rangle .
\end{aligned}
$$

Regarding the $z_{1}$ dependence of $V_{02}(z)$, use $(9)$ to get

$$
L_{V_{0}} \hat{c}(z)=\left\langle x_{1}, z_{1}\right\rangle\left\langle x_{1}, z_{2}\right\rangle=\left(x_{1} \otimes x_{1}\right)\left(z_{1}, z_{2}\right)=\hat{c}^{\prime}(z) .
$$

Applying (8) and (9) a second time gives:

$$
\begin{aligned}
L_{V_{0}} L_{V_{0}} \hat{c}(z) & =L_{V_{0}} \hat{c}^{\prime}(z) \\
& =\left\langle x_{1}, V_{01}(z) z_{1}\right\rangle\left\langle x_{1}, z_{2}\right\rangle+\left\langle x_{1}, z_{1}\right\rangle\left\langle x_{1}, V_{02}(z) z_{2}\right\rangle \\
& =\left\langle x_{1}, x_{0} z_{1}\right\rangle\left\langle x_{1}, z_{2}\right\rangle+\left\langle x_{1}, z_{1}\right\rangle\left\langle x_{1},\left(x_{0}+x_{1}\left\langle x_{1}, z_{1}\right\rangle\right) z_{2}\right\rangle \\
& =\left\langle x_{1}, z_{1}\right\rangle^{2}\left\langle\mathbf{1}, z_{2}\right\rangle \\
& =\left\langle x_{1} \amalg x_{1}, z_{1}\right\rangle\left\langle\mathbf{1}, z_{2}\right\rangle \\
& =\left(x_{1} \amalg x_{1} \otimes \mathbf{1}\right)\left(z_{1}, z_{2}\right) \\
& =\left(2 x_{1}^{2} \otimes \mathbf{1}\right)\left(z_{1}, z_{2}\right)=\hat{c}^{\prime \prime}(z) .
\end{aligned}
$$

Continuing in this fashion,

$$
\begin{aligned}
L_{V_{1}} L_{V_{0}} L_{V_{0}} \hat{c}(z) & =L_{V_{1}} \hat{c}^{\prime \prime}(z)=\left\langle 2 x_{1}, z_{1}\right\rangle\left\langle\mathbf{1}, z_{2}\right\rangle \\
& =\left(2 x_{1} \otimes \mathbf{1}\right)\left(z_{1}, z_{2}\right)=\hat{c}^{\prime \prime \prime}(z)
\end{aligned}
$$

and

$$
L_{V_{1}} L_{V_{1}} L_{V_{0}} L_{V_{0}} \hat{c}(z)=L_{V_{1}} \hat{c}^{\prime \prime \prime}(z)=\left\langle 2 \mathbf{1}, z_{1}\right\rangle\left\langle\mathbf{1}, z_{2}\right\rangle .
$$

Therefore, $\left\langle x_{0}^{2} x_{1}^{2}, c \circ d\right\rangle=L_{V_{1}} L_{V_{1}} L_{V_{0}} L_{V_{0}} \hat{c}(\mathbf{1})=2$ as anticipated. 
The proposition in the previous example is established in the general case by the following theorem.

Theorem 4.1. If $d \in \mathbb{R}\langle\langle X\rangle\rangle$ has a well defined formal representation $\left(\mu, z_{0}, \hat{c}_{k}\right)$, then the input-output map $u \mapsto y_{k}$ of the corresponding formal realization (10)-(11) has a Chen-Fliess series representation with generating series $d$.

Proof: Without loss of generality, assume there is a single output so that the subscripts on $\hat{c}_{k}$ and $y_{k}$ can be dropped. Likewise, assume $n=1$ so the index on the state can be omitted. Since $\dot{z}(t)$ is a tangent vector at $z(t) \in \mathcal{G}(X)$ for any $t \geq 0$, it follows directly from (7) that

$$
\begin{aligned}
\dot{z}(t)(\hat{c}) & =\sum_{i=0}^{m} V_{i}(z(t))(\hat{c}) u_{i}(t) \\
& =\sum_{i=0}^{m} L_{V_{i}} \hat{c}(z(t)) u_{i}(t) .
\end{aligned}
$$

Integrating both sides on $[0, t]$ and applying $(9)$ gives

$$
\begin{aligned}
\hat{c}(z(t)) & =\hat{c}\left(z_{0}\right)+\sum_{i=0}^{m} \int_{0}^{t} L_{V_{i}} \hat{c}(z(\tau)) u_{i}(\tau) d \tau \\
& =\hat{c}\left(z_{0}\right)+\sum_{i=0}^{m} \int_{0}^{t} \hat{c}_{i}^{\prime}(z(\tau)) u_{i}(\tau) d \tau
\end{aligned}
$$

where $L_{V_{i}} \hat{c}(z(\tau))=\hat{c}_{i}^{\prime}(z(\tau))=\left\langle\hat{c}_{i}^{\prime}, z(\tau)\right\rangle$. Substituting $\hat{c}_{i}^{\prime}$ for $\hat{c}$ above yields

$$
\hat{c}_{i}^{\prime}(z(t))=\hat{c}_{i}^{\prime}\left(z_{0}\right)+\sum_{i=0}^{m} \int_{0}^{t} \hat{c}_{i}^{\prime \prime}(z(\tau)) u_{i}(\tau) d \tau .
$$

Noting that $y(t)=\hat{c}(z(t))$ and substituting 14 into (13) gives

$$
\begin{aligned}
y(t)= & \hat{c}\left(z_{0}\right)+\sum_{i=0}^{m} L_{V_{i}} \hat{c}\left(z_{0}\right) \int_{0}^{t} u_{i}(\tau) d \tau+ \\
& \sum_{i_{1}, i_{2}=0}^{m} \int_{0}^{t} \int_{0}^{\tau_{1}} L_{V_{i_{1}}} \hat{c}_{i_{2}}\left(z\left(\tau_{2}\right)\right) u_{i_{2}}\left(\tau_{2}\right) d \tau_{2} u_{i_{1}}\left(\tau_{1}\right) d \tau_{1} .
\end{aligned}
$$

Continuing in this way yields

$$
\begin{aligned}
y(t) & =\sum_{\eta \in X^{*}} L_{\mu(\eta)} \hat{c}\left(z_{0}\right) E_{\eta}[u](t) \\
& =\sum_{\eta \in X^{*}}\langle d, \eta\rangle E_{\eta}[u](t)
\end{aligned}
$$

which proves the theorem. 


\section{Networks of Chen-Fliess Series}

In this section specific types of networks of Chen-Fliess series are considered for which both Lemma 4.1 and Theorem 4.1 apply. To avoid a barrage of indices, the component systems are assumed to be single-input, single-output. There is, however, no technical reason for avoiding the multivariable case. A variety of different configurations are possible. The following is perhaps the simplest.

Definition 5.1. A set of $m$ single-input, single-output Chen-Fliess series mapping $u_{i} \mapsto y_{i}$ with generating series $c_{i} \in \mathbb{R}_{L C}\left\langle\left\langle X_{i}\right\rangle\right\rangle$, where $X_{i}=\left\{x_{0}, x_{i}\right\}$, and weighting matrix $M \in \mathbb{R}^{m \times m}$ is said to be additively interconnected if $u_{i}=v_{i}+\sum_{j=1}^{m} M_{i j} y_{j}, i=1,2, \ldots, m$.

In the following theorem, let $\boldsymbol{e}_{i} \in \mathbb{R}_{L C}^{m}\langle\langle X\rangle\rangle$ denote the series with the $i$-th component series being the monomial $\mathbf{1}$, and the remaining components are the series having all coefficients equal to zero. In addition, given $c_{j} \in \mathbb{R}_{L C}\langle\langle X\rangle\rangle$, define $\hat{c}_{j}=\mathbf{1} \otimes \cdots \otimes \mathbf{1} \otimes c_{j} \otimes \mathbf{1} \cdots \otimes \mathbf{1} \in$ $\mathbb{R}_{L C}^{\otimes m}\langle\langle X\rangle\rangle$, where $c_{j}$ appears in the $j$-th position.

Theorem 5.1. The input-output map $v \mapsto y$ of any additive interconnection of $m$ singleinput, single-output Chen-Fliess series with generating series $c_{i} \in \mathbb{R}_{L C}\left\langle\left\langle X_{i}\right\rangle\right\rangle$ has a well defined generating series $d \in \mathbb{R}^{m}\langle\langle X\rangle\rangle$, where $d_{j}$ has the formal representation $\left(\mu, \mathbf{1}_{m}, \hat{c}_{j}\right)$ with $\mu$ defined in terms of the vector fields

$$
V_{0}(z)=\left[\begin{array}{c}
x_{0} z_{1} \\
x_{0} z_{2} \\
\vdots \\
x_{0} z_{m}
\end{array}\right]+\operatorname{diag}\left(x_{1} z_{1}, \ldots, x_{m} z_{m}\right) M\left[\begin{array}{c}
\left\langle c_{1}, z_{1}\right\rangle \\
\left\langle c_{2}, z_{2}\right\rangle \\
\vdots \\
\left\langle c_{m}, z_{m}\right\rangle
\end{array}\right],
$$

and $V_{i}(z)=x_{i} z_{i} \boldsymbol{e}_{i}$ for $i=1,2, \ldots, m$.

Proof: It is straightforward to show that the set of interconnected Chen-Fliess series constitutes an $m$ input, $m$ output system with formal realization given by the vector fields as shown. Therefore, the claim follows directly from Lemma 4.1 and Theorem 4.1 with $\mu\left(x_{i}\right)=V_{i}, i=0,1, \ldots m, z_{0}=\mathbf{1}_{m}$, and $\hat{c}_{j} \in \mathbb{R}_{L C}^{\otimes m}\langle\langle X\rangle\rangle$.

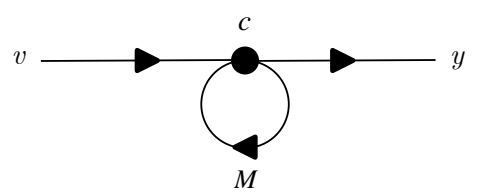

FiguRE 1. Single system additively interconnected

Example 5.1. A single system additively interconnected with itself as shown in Figure 1 would correspond to propositional output feedback, i.e., $u=v+M y$ (dropping all subscripts). Thus, the corresponding representation is given by

$$
V_{0}(z)=\left(x_{0}+x_{1} M\langle c, z\rangle\right) z, \quad V_{1}(z)=x_{1} z,
$$


$z_{0}=\mathbf{1}_{1}=\mathbf{1}$, and $\hat{c}=c$. For a unity feedback system, i.e., $M=1$, applying $(12)$ gives the following generating series for the closed-loop system:

$$
\begin{aligned}
\langle d, \mathbf{1}\rangle= & c(\mathbf{1})=\langle c, \mathbf{1}\rangle \\
\left\langle d, x_{1}\right\rangle= & L_{V_{1}} c(\mathbf{1})=\left\langle c, x_{1}\right\rangle \\
\left\langle d, x_{0}\right\rangle= & L_{V_{0}} c(\mathbf{1})=\left\langle c, x_{0}\right\rangle+\left\langle c, x_{1}\right\rangle\langle c, \mathbf{1}\rangle \\
\left\langle d, x_{1}^{2}\right\rangle= & L_{V_{1}} L_{V_{1}} c(\mathbf{1})=\left\langle c, x_{1}^{2}\right\rangle \\
\left\langle d, x_{0} x_{1}\right\rangle= & L_{V_{1}} L_{V_{0}} c(\mathbf{1})=\left\langle c, x_{0} x_{1}\right\rangle+\left\langle c, x_{1}\right\rangle\left\langle c, x_{1}\right\rangle+\left\langle c, x_{1}^{2}\right\rangle\langle c, \mathbf{1}\rangle \\
\left\langle d, x_{1} x_{0}\right\rangle= & L_{V_{0}} L_{V_{1}} c(\mathbf{1})=\left\langle c, x_{1} x_{0}\right\rangle+\left\langle c, x_{1}^{2}\right\rangle\langle c, \mathbf{1}\rangle \\
\left\langle d, x_{0}^{2}\right\rangle= & L_{V_{0}} L_{V_{0}} c(\mathbf{1})=\left\langle c, x_{0}^{2}\right\rangle+\left\langle c, x_{1}\right\rangle\left\langle c, x_{0}\right\rangle+\left\langle c, x_{1} x_{0}\right\rangle\langle c, \mathbf{1}\rangle+ \\
& \left\langle c, x_{0} x_{1}\right\rangle\langle c, \mathbf{1}\rangle+\left\langle c, x_{1}\right\rangle\left\langle c, x_{1}\right\rangle\langle c, \mathbf{1}\rangle+\left\langle c, x_{1}^{2}\right\rangle\langle c, \mathbf{1}\rangle\langle c, \mathbf{1}\rangle
\end{aligned}
$$

These expressions are consistent with those in [7], where $d=S(-c)$, and $S$ is the antipode of the output feedback Hopf algebra.

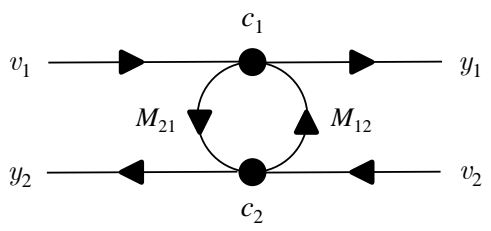

FigurE 2. Two systems additively interconnected

Example 5.2. Consider two additively interconnected systems as shown in Figure 2, where $M_{i j}=0$ when $i=j$. Setting $M_{i j}=1$ for $i \neq j$ gives a representation of $d_{j}$ specified by

$$
V_{0}(z)=\left[\begin{array}{c}
\left(x_{0}+x_{1}\left\langle c_{2}, z_{2}\right\rangle\right) z_{1} \\
\left(x_{0}+x_{2}\left\langle c_{1}, z_{1}\right\rangle\right) z_{2}
\end{array}\right], \quad V_{i}(z)=x_{i} z_{i} \boldsymbol{e}_{i}, \quad i=1,2,
$$

$z_{0}=\mathbf{1}_{2}$, and $\hat{c}_{j}$. For example, the generating series $d_{1}$ for the mapping $v \mapsto y_{1}$ is:

$$
\begin{aligned}
\left\langle d_{1}, \mathbf{1}\right\rangle & =\hat{c}_{1}\left(\mathbf{1}_{2}\right)=\left\langle c_{1}, \mathbf{1}\right\rangle \\
\left\langle d_{1}, x_{1}\right\rangle & =L_{V_{1}} \hat{c}_{1}\left(\mathbf{1}_{2}\right)=\left\langle c_{1}, x_{1}\right\rangle \\
\left\langle d_{1}, x_{2}\right\rangle & =L_{V_{2}} \hat{c}_{1}\left(\mathbf{1}_{2}\right)=0 \\
\left\langle d_{1}, x_{0}\right\rangle & =L_{V_{0}} \hat{c}_{1}\left(\mathbf{1}_{2}\right)=\left\langle c_{1}, x_{0}\right\rangle+\left\langle c_{1}, x_{1}\right\rangle\left\langle c_{2}, \mathbf{1}\right\rangle \\
\left\langle d_{1}, x_{1}^{2}\right\rangle & =L_{V_{1}} L_{V_{1}} \hat{c}_{1}\left(\mathbf{1}_{2}\right)=\left\langle c_{1}, x_{1}^{2}\right\rangle \\
\left\langle d_{1}, x_{1} x_{2}\right\rangle & =L_{V_{2}} L_{V_{1}} \hat{c}_{1}\left(\mathbf{1}_{2}\right)=0 \\
\left\langle d_{1}, x_{2} x_{1}\right\rangle & =L_{V_{1}} L_{V_{2}} \hat{c}_{1}\left(\mathbf{1}_{2}\right)=0 \\
\left\langle d_{1}, x_{2}^{2}\right\rangle & =L_{V_{2}} L_{V_{2}} \hat{c}_{1}\left(\mathbf{1}_{2}\right)=0 \\
\left\langle d_{1}, x_{1} x_{0}\right\rangle & =L_{V_{0}} L_{V_{1}} \hat{c}_{1}\left(\mathbf{1}_{2}\right)=\left\langle c_{1}, x_{1} x_{0}\right\rangle+\left\langle c_{1}, x_{1}^{2}\right\rangle\left\langle c_{2}, \mathbf{1}\right\rangle
\end{aligned}
$$




$$
\left\langle d_{1}, x_{0} x_{1}\right\rangle=L_{V_{1}} L_{V_{0}} \hat{c}_{1}\left(\mathbf{1}_{2}\right)=\left\langle c_{1}, x_{0} x_{1}\right\rangle+\left\langle c_{1}, x_{1}^{2}\right\rangle\left\langle c_{2}, \mathbf{1}\right\rangle
$$

and similarly for $d_{2}$ corresponding to the map $v \mapsto y_{2}$. Unlike the first example, for networks with more than one system, there is at present no known alterative algebraic method against which to compare all of these results. Coefficient $\left\langle d_{1}, \eta\right\rangle$, where $\eta \in X_{j}^{*}$ and $j=1,2$ can be determined using the feedback product as described in [7], but mixed coefficients like $\left\langle d_{1}, x_{1} x_{2}\right\rangle$ can not.

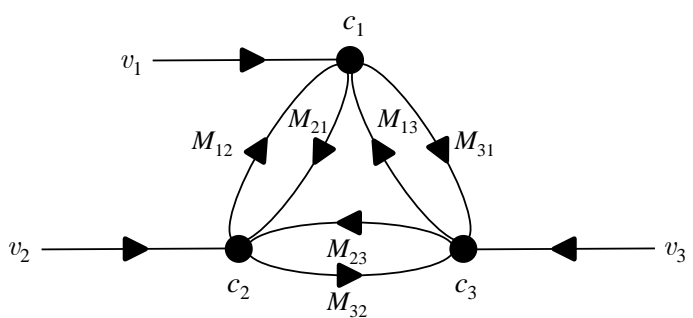

FIGURE 3. Three systems additively interconnected

Example 5.3. Consider three additively interconnected systems as shown in Figure 3, where again $M_{i j}=0$ when $i=j$, and the output branches have been suppressed. For the case where $M_{i j}=1$ when $i \neq j$, a representation of $d_{j}$ is given by

$$
\begin{aligned}
& V_{0}(z)=\left[\begin{array}{c}
\left(x_{0}+x_{1}\left\langle c_{2}, z_{2}\right\rangle+x_{1}\left\langle c_{3}, z_{3}\right\rangle\right) z_{1} \\
\left(x_{0}+x_{2}\left\langle c_{1}, z_{1}\right\rangle+x_{2}\left\langle c_{3}, z_{3}\right\rangle\right) z_{2} \\
\left(x_{0}+x_{3}\left\langle c_{1}, z_{1}\right\rangle+x_{3}\left\langle c_{2}, z_{2}\right\rangle\right) z_{3}
\end{array}\right] \\
& V_{i}(z)=x_{i} z_{i} \boldsymbol{e}_{i}, \quad i=1,2,3,
\end{aligned}
$$

$z_{0}=\mathbf{1}_{3}$, and $\hat{c}_{j}$. For example, the generating series $d_{1}$ for the mapping $v \mapsto y_{1}$ is:

$$
\begin{aligned}
\left\langle d_{1}, \mathbf{1}\right\rangle & =\hat{c}_{1}\left(\mathbf{1}_{3}\right)=\left\langle c_{1}, \mathbf{1}\right\rangle \\
\left\langle d_{1}, x_{1}\right\rangle & =L_{V_{1}} \hat{c}_{1}\left(\mathbf{1}_{3}\right)=\left\langle c_{1}, x_{1}\right\rangle \\
\left\langle d_{1}, x_{2}\right\rangle & =L_{V_{2}} \hat{c}_{1}\left(\mathbf{1}_{3}\right)=0 \\
\left\langle d_{1}, x_{3}\right\rangle & =L_{V_{3}} \hat{c}_{1}\left(\mathbf{1}_{3}\right)=0 \\
\left\langle d_{1}, x_{0}\right\rangle & =L_{V_{0}} \hat{c}_{1}\left(\mathbf{1}_{3}\right)=\left\langle c_{1}, x_{0}\right\rangle+\left\langle c_{1}, x_{1}\right\rangle\left\langle c_{2}, \mathbf{1}\right\rangle+\left\langle c_{1}, x_{1}\right\rangle\left\langle c_{3}, \mathbf{1}\right\rangle \\
\left\langle d_{1}, x_{1}^{2}\right\rangle & =L_{V_{1}} L_{V_{1}} \hat{c}_{1}\left(\mathbf{1}_{3}\right)=\left\langle c_{1}, x_{1}^{2}\right\rangle \\
\left\langle d_{1}, x_{1} x_{2}\right\rangle & =L_{V_{2}} L_{V_{1}} \hat{c}_{1}\left(\mathbf{1}_{3}\right)=0 \\
\left\langle d_{1}, x_{1} x_{3}\right\rangle & =L_{V_{3}} L_{V_{1}} \hat{c}_{1}\left(\mathbf{1}_{3}\right)=0 \\
\left\langle d_{1}, x_{1} x_{0}\right\rangle & =L_{V_{0}} L_{V_{1}} \hat{c}_{1}\left(\mathbf{1}_{3}\right)=\left\langle c_{1}, x_{1} x_{0}\right\rangle+\left\langle c_{1}, x_{1}^{2}\right\rangle\left\langle c_{2}, \mathbf{1}\right\rangle+\left\langle c_{1}, x_{1}^{2}\right\rangle\left\langle c_{3}, \mathbf{1}\right\rangle \\
\left\langle d_{1}, x_{0} x_{1}\right\rangle & =L_{V_{1}} L_{V_{0}} \hat{c}_{1}\left(\mathbf{1}_{3}\right)=\left\langle c_{1}, x_{0} x_{1}\right\rangle+\left\langle c_{1}, x_{1}^{2}\right\rangle\left\langle c_{2}, \mathbf{1}\right\rangle+\left\langle c_{1}, x_{1}^{2}\right\rangle\left\langle c_{3}, \mathbf{1}\right\rangle
\end{aligned}
$$

and similarly for $d_{i}$ corresponding to the map $v \mapsto y_{i}, i=2,3$. 
Free from the bonds of linearity, other types of interconnections are also possible as considered next.

Definition 5.2. A set of $m$ single-input, single-output Chen-Fliess series mapping $u_{i} \mapsto y_{i}$ with generating series $c_{i} \in \mathbb{R}_{L C}\left\langle\left\langle X_{i}\right\rangle\right\rangle$, where $X_{i}=\left\{x_{0}, x_{i}\right\}$, and weighting matrix $M \in \mathbb{R}^{m \times m}$ is said to be multiplicatively interconnected if $u_{i}=v_{i} \prod_{j=1}^{m} M_{i j} y_{j}, i=1,2, \ldots, m$.

Theorem 5.2. Every input-output map $v \mapsto y$ of any multiplicative interconnection of $m$ single-input, single-output Chen-Fliess series with generating series $c_{i} \in \mathbb{R}_{L C}\left\langle\left\langle X_{i}\right\rangle\right\rangle$ has a well defined generating series $d \in \mathbb{R}^{m}\langle\langle X\rangle\rangle$, where $d_{j}$ has the formal representation $\left(\mu, \mathbf{1}_{m}, \hat{c}_{j}\right)$ with $\mu$ defined in terms of the vector fields

$$
V_{0}(z)=\left[\begin{array}{c}
x_{0} z_{1} \\
x_{0} z_{2} \\
\vdots \\
x_{0} z_{m}
\end{array}\right], \quad V_{i}(z)=x_{i} \prod_{j=1}^{m} M_{i j}\left\langle c_{j}, z_{j}\right\rangle z_{i} \boldsymbol{e}_{i}
$$

Proof: The proof is perfectly analogous to that of Theorem 5.1 .

Example 5.4. Reconsider the single system network in Example 5.1 except now multiplicatively interconnected, that is, $u=v M y$ (again dropping all subscripts). The corresponding representation is given by

$$
V_{0}(z)=x_{0} z, \quad V_{1}(z)=x_{1} M\langle c, z\rangle z,
$$

$z_{0}=\mathbf{1}$, and $\hat{c}=c$. Setting $M=1$ and applying $(12)$ gives the following generating series for the closed-loop system:

$$
\begin{aligned}
\langle d, \mathbf{1}\rangle= & c(\mathbf{1})=\langle c, \mathbf{1}\rangle \\
\left\langle d, x_{1}\right\rangle= & L_{V_{1}} c(\mathbf{1})=\left\langle c, x_{1}\right\rangle\langle c, \mathbf{1}\rangle \\
\left\langle d, x_{0}\right\rangle= & L_{V_{0}} c(\mathbf{1})=\left\langle c, x_{0}\right\rangle \\
\left\langle d, x_{1}^{2}\right\rangle= & L_{V_{1}} L_{V_{1}} c(\mathbf{1})=\left\langle c, x_{1}^{2}\right\rangle\langle c, \mathbf{1}\rangle\langle c, \mathbf{1}\rangle+\left\langle c, x_{1}\right\rangle\left\langle c, x_{1}\right\rangle\langle c, \mathbf{1}\rangle \\
\left\langle d, x_{0} x_{1}\right\rangle= & L_{V_{1}} L_{V_{0}} c(\mathbf{1})=\left\langle c, x_{0} x_{1}\right\rangle\langle c, \mathbf{1}\rangle \\
\left\langle d, x_{1} x_{0}\right\rangle= & L_{V_{0}} L_{V_{1}} c(\mathbf{1})=\left\langle c, x_{1} x_{0}\right\rangle\langle c, \mathbf{1}\rangle+\left\langle c, x_{1}\right\rangle\left\langle c, x_{0}\right\rangle \\
\left\langle d, x_{1}^{3}\right\rangle= & L_{V_{1}} L_{V_{1}} L_{V_{1}} c(\mathbf{1})=\left\langle c, x_{1}^{3}\right\rangle\langle c, \mathbf{1}\rangle\langle c, \mathbf{1}\rangle\langle c, \mathbf{1}\rangle+4\left\langle c, x_{1}^{2}\right\rangle\left\langle c, x_{1}\right\rangle\langle c, \mathbf{1}\rangle\langle c, \mathbf{1}\rangle+ \\
& \left\langle c, x_{1}\right\rangle\left\langle c, x_{1}\right\rangle\left\langle c, x_{1}\right\rangle\langle c, \mathbf{1}\rangle \\
\left\langle d, x_{0}^{2}\right\rangle= & L_{V_{0}} L_{V_{0}} c(\mathbf{1})=\left\langle c, x_{0}^{2}\right\rangle
\end{aligned}
$$

Consider the particular case where $c=\sum_{k \geq 0} k ! x_{1}^{k}$. Applying the formulas above gives the closed-loop generating series

$$
d=1+x_{1}+3 x_{1}^{2}+15 x_{1}^{3}+\cdots,
$$


which is consistent with what was computed in [8, Example 4.10] using the antipode of the output affine feedback Hopf algebra.

\section{Conclusions and Future Work}

Using the concept of a formal realization and a formal representation, it was shown that any additive or multiplicative interconnection of a set of convergent single-input, singleoutput Chen-Fliess series always has a Chen-Fliess series representation whose generating series can be computed explicitly in terms of iterated formal Lie derivatives. This of course does not exhaust the list of possible network topologies for which this method is suitable. For example, there can be mixtures of additive and multiplicative nodes in a given network. There is also no technical barrier to applying the methodology in the full multivariable setting. Finally, the issue of convergence of the network's generating series needs to be addressed in every case.

\section{ACKNOWLEDGMENTS}

The first author was supported by the National Science Foundation under grant CMMI1839378. The second author was supported by the Research Council of Norway through project 302831 Computational Dynamics and Stochastics on Manifolds (CODYSMA).

\section{REFERENCES}

[1] J. Berstel and C. Reutenauer, Rational Series and Their Languages, Springer, Berlin, 1988.

[2] P. Cartier, Démonstration algébrique de la formule de Hausdorff, Bull. Soc. Math. France, 84 (1956) 241-249.

[3] G. Casadei, D. Astolfi, A. Alessandri, and L. Zaccarian, Synchronization in networks of identical nonlinear systems via dynamic dead zones, IEEE Control Systems Lett., 3 (2019) 667-672.

[4] A. Ferfera, Combinatoire du monoïde libre et composition de certains systèmes non linéaires, Astérisque, 75-76 (1980) 87-93.

[5] M. Fliess, Fonctionnelles causales non linéaires et indéterminées non commutatives, Bull. Soc. Math. France, 109 (1981) 3-40.

[6] M. Fliess, Réalisation locale des systèmes non linéaires, algèbres de Lie filtrées transitives et séries génératrices non commutatives, Invent. Math., 71 (1983) 521-537.

[7] W. S. Gray, L. A. Duffaut Espinosa, and K. Ebrahimi-Fard, Faà di Bruno Hopf algebra of the output feedback group for multivariable Fliess operators, Systems Control Lett., 74 (2014) 64-73.

[8] W. S. Gray and K. Ebrahimi-Fard, SISO output affine feedback transformation group and its Faà di Bruno Hopf algebra, SIAM J. Control Optim., 55 (2017) 885-912.

[9] W. S. Gray, G. S. Venkatesh, and L. A. Duffaut Espinosa, Nonlinear system identification for multivariable control via discrete-time Chen-Fliess series, Automatica, 119 (2020) 109085.

[10] W. S. Gray and Y. Wang, Fliess operators on $L_{p}$ spaces: Convergence and continuity, Systems Control Lett., 46 (2002) 67-74.

[11] R. Grossman and R. G. Larson, The realization of input-output maps using bialgebras, Forum Mathematicum, 4 (1992) 109-121.

[12] L. Grunenfelder, Algebraic aspects of control systems and realizations, J. Algebra, 165 (1994) 446-464.

[13] A. Isidori, Nonlinear Control Systems, 3rd Ed., Springer, London, 1995. 
[14] M. Kawski and H. J. Sussmann, Noncommutative power series and formal Lie-algebraic techniques in nonlinear control theory, in Operators, Systems, and Linear Algebra: Three Decades of Algebraic Systems Theory, U. Helmke, D. Prätzel-Wolters, and E. Zerz, Eds., B. G. Teubner, Stuttgart, 1997, pp. 111-128.

[15] D. Manchon, Hopf algebras and renormalisation, in Handbook of Algebra, vol. 5, M. Hazewinkel, Ed., Elsevier B.V. (North-Holland), Amsterdam, 2008, 365-427.

[16] H. Nijmeijer and A. J. van der Schaft, Nonlinear Dynamical Control Systems, Springer, New York, 1990.

[17] R. Ree, Lie elements and an algebra associated with shuffles, Ann. of Math. (2), 68 (1958) 210-220.

[18] C. Reutenauer, Free Lie Algebras, Oxford University Press, New York, 1993.

[19] H. J. Sussmann, A product expansion for the Chen series, in Theory and Applications of Nonlinear Control Systems, C. I. Byrnes and A. Lindquist, Eds., Elsevier Science Publishers B.V. (North Holland), New York, 1986, pp. 323-335.

[20] M. Thitsa and W. S. Gray, On the radius of convergence of interconnected analytic nonlinear inputoutput systems, SIAM J. Control Optim., 50 (2012) 2786-2813.

[21] G. S. Venkatesh, W. S. Gray, and L. A. Duffaut Espinosa, Combining learning and model based multivariable control, Proc. 58th IEEE Conf. on Decision and Control, Nice, France, 2019, pp. 1013-1018.

[22] Y. Wang, Differential Equations and Nonlinear Control Systems, Doctoral Dissertation, Rutgers University, New Brunswick, NJ, 1990.

[23] A. J. Whalen, S. N. Brennan, T. D. Sauer, and S. J. Schiff, Observability and controllability of nonlinear networks: The role of symmetry, Phys. Rev. X, 5 (2015) 011005.

Department of Electrical and Computer Engineering, Old Dominion University, NorFOLK, ViRginia 23529, USA

Email address: sgray@odu.edu

URL: http://www.ece.odu.edu/ sgray/

Department of Mathematical Sciences, Norwegian University of Science and Technology (NTNU), 7491 Trondheim, Norway

Email address: kurusch.ebrahimi-fard@ntnu.no

$U R L$ : https://folk.ntnu.no/kurusche/ 